\title{
Incentivizing Safer Sexual Behavior: Evidence from a Lottery Experiment on HIV Prevention
}

\author{
By Martina BJörkman Nyqvist, Lucia Corno, Damien de Walque, And \\ JAKOB SVENSSON*
}

\begin{abstract}
We investigate the effect of a financial lottery program in Lesotho with relatively low expected payments but a chance to win a high prize conditional on negative test results for sexually transmitted infections. The intervention resulted in a 21.4 percent reduction in HIV incidence over two years. Lottery incentives appear to be particularly effective in targeting individuals with ex ante risky sexual behavior, consistent with the hypothesis that lotteries are more valued by individuals willing to take risks.
\end{abstract}

JEL: $112,115,015$

Keywords: Financial incentives, Lotteries, HIV prevention

\section{Introduction}

An estimated 1.2 million new HIV infections occurred in Africa alone in 2016, adding to the nearly 26 million people living with HIV on the continent. New infections continue to outpace antiretroviral therapy uptake in most developing countries (UNAIDS, 2016). Thus the global need for effective HIV prevention programs remains urgent.

In this paper we present the results from a financial incentive program designed in the form of a lottery with low expected payments ( $\$ 20-\$ 40$ per year) conditional on testing negative for two markers of risky sexual behaviors (two curable sexually transmitted infections, STI), but with relatively high payments for lottery winners.

The use of lotteries in public health interventions is not unprecedented, including in

* Björkman Nyqvist: Stockholm School of Economics, Department of Economics, Sveavägen 65, 11383 Stockholm, Sweden (martina.bjorkman.nyqvist@hhs.se). Corno: Università Cattolica del Sacro Cuore, Department of Economics and Finance, Largo Gemelli 1, Milan, Italy, lucia.corno@unicatt.it. de Walque: Development Research Group, The World Bank, 1818 H street NW, Washington DC 20433, USA, ddewalque @ worldbank.org. Svensson: Institute for International Economic Studies (IIES), Stockholm University, 10691 Stockholm, Sweden (jakob.svensson@iies.su.se). We gratefully acknowledge four anonymous referees for valuable comments and suggestions. We are grateful to the Ministry of Health and Social Welfare in Lesotho, the World Bank Lesotho Office, Population Service International and Fidelis Ensejor of Leads Consultancy for excellent collaboration. We thank Imran Rasul, David Strömberg, Tessa Bold, Erik Lindqvist, Robert Östling, David McKenzie and participants at seminars at Institute of Fiscal Studies; Bocconi University; London School of Hygiene and Tropical Medicine; Stockholm University; University of Bristol; IFPRI, LACEA, PopPov, International AIDS Conference. Sara Berman, Serena Cocciolo, Francesca Grazioli, Elisa Maffioli, Mattia Chiapello, and Arianna Ornaghi provided excellent research assistance. Financial support from the World Bank's Strategic Impact Evaluation Fund, Bank-Netherlands Partnership Program, Trust Fund for Environmentally \& Socially Sustainable Development and Knowledge for Change Program, the Swedish Research Council (421-2009-2209), the Program for Development Research, SIDA, and from Swedish Foundation for Humanities and Social Sciences is gratefully acknowledged. The findings, interpretations, and conclusions expressed in this paper are entirely those of the authors. They do not necessarily represent the views of the World Bank, its Executive Directors, or the countries they represent. The study was registered at the American Economic Association Registry for Randomized Controlled Trials (RCTs): the trial registration number is AEARCTR-0000567. 
the area of HIV prevention. ${ }^{1}$ To the best of our knowledge, however, this is the first large scale randomized trials to assess its impact as a prevention tool. ${ }^{2}$ Introducing a gamble into an otherwise standard financial incentive program, such as a conditional cash transfer, has at least two potential advantages. First, with lotteries, the program becomes relatively more attractive to individuals that are willing to take monetary risks. If individuals exhibiting risk-loving preferences in monetary gambles are also more riskloving in other domains, including sexual behavior, lottery incentives may better target those at higher risk of HIV/AIDS. Second, there is growing evidence from psychology and behavioral economics that people tend to overestimate small percentages, and therefore prefer a small chance at a large reward to a small reward for sure (Kahneman and Tversky, 1979, Kahneman, 2011, Barberis, 2013). If so, the perceived return from participating in a lottery may be higher than the return from an incentive program that pays the expected return with certainty, or likewise, lotteries may provide stronger incentives for behavioral change compared to a traditional conditional cash transfer.

We find that HIV incidence in the intervention groups fell by 21.4 percent relative to the control group over a two-year period and estimate that the cost of averting a primary HIV infection was $\$ 882$, or about one-tenth of the costs of averting an HIV infection in the only other behavioral trial documenting significant reduction in HIV prevalence (Baird et al., 2012). ${ }^{3}$ Consistent with the reduction in risky sexual behavior, we also document large reductions in both the prevalence of STI and in births and pregnancies, especially among single women.

Despite the lack of a certainty equivalent intervention arm, we provide suggestive evidence that the lottery design played an important role in achieving the effects we document. Using baseline data from the control group, we show that risk-lovers - with the degree of risk aversion measured using a simple multiple price list design - were more than twice as likely to become infected with HIV over the trial period, thus providing support for the assumption that risk-loving behavior in hypothetical monetary gambles translates to objectively measured risk-loving sexual behavior. In the intervention groups, on the other hand, where participants were offered lottery incentives to promote safer sexual behavior, we find that the risk-loving individuals were as likely as risk-averse individu-

\footnotetext{
${ }^{1}$ The HIV screening lottery in the Western Cape province in South Africa is probably the most notable example. The lottery, developed as a pilot by a Harvard-affiliated organization set up to develop psychology and economics-based strategies for social policy - aims at encouraging people to get tested for HIV (Keating, 2013). Another well-known example of the use of lotteries in public health programs is the 1957 Tuberculosis-screening campaign implemented in Glasgow. Geffen (2011) reports that intense media coverage and a weekly prize draw resulted in a number of screenings almost three times higher than the initial aim of reaching 250,000 people.

${ }^{2}$ Recent trials have used lottery incentives to improve adherence to blood thinners (Volpp et al., 2008b; Kimmel et al., 2016) and to a 6-month vaccination protocol (Stitzer et al., 2010). They generally find that lottery incentives led to an improvement in the adherence measures compared to the control group. Two US studies compare lottery incentives with some type of fixed payment incentives. Volpp et al. (2008a) compare lottery incentives and a deposit or commitment device contract whereby participants pledge upfront their own money as incentives for weight loss. During the 16 weeks incentive period, both incentives led to similar reductions in weight compared to the control group, even though the average amount of money earned was not similar across the two groups, but those effects were not sustained at a seven months weigh-in. Haisley et al. (2012) compare lottery incentives combined with a group-level incentive with the certainty equivalent (of the lottery incentives) incentives in an intervention aimed at encouraging members of an organization to complete individual health risk assessments. The lottery and group level incentives outperformed the fixed payment incentives and the control group.

${ }^{3} \mathrm{HIV}$ incidence is the flow of new infections; HIV prevalence is the stock of existing infections.
} 
als to become HIV infected during the two-year trial period. That is, the lottery program lowered HIV incidence rates in the treatment relative to the control group by making individuals with greater preference for risk at baseline behave similarly to risk-averse individuals in terms of their sexual behavior.

Our paper relates to the growing literature on prevention programs for HIV/AIDS. Such programs can be broadly divided into two types: biomedical interventions that aim at reducing susceptibility to HIV, holding behavior constant, and interventions aimed at reducing risky sexual behavior. Several biomedical interventions, such as male circumcision and pre-exposure prophylaxis, have been shown to significantly reduce HIV transmission in field trials (Gray et al., 2007; Bailey et al., 2007; and Auvert et al., 2005). Evidence from randomized controlled trials of behavioral interventions, however, is at best mixed (see McCoy et al. 2010 and Padian et al. 2010 for reviews). No traditional programs offering some combination of risk-reduction counseling, condom promotion, referral and treatment for sexually transmitted infections have documented significant effects on HIV incidence. More recent evidence has shown that financial incentive interventions aimed at promoting safe sexual behavior by conditional payments on HIV or STI status, or school enrollment, can have an impact, at least when the financial payments are sufficiently large. For example, Baird et al. (2012) evaluate an intervention where cash transfers ( $\$ 120$ per year and household) were conditional on school attendance for adolescent girls. The intervention led to a significant reduction in HIV prevalence after 18 months. De Walque et al. (2012) evaluate a program in Tanzania where cash transfers were made conditional on remaining negative for a set of curable STI. They document a significant reduction in STI prevalence in the intervention arm where participants were eligible for $\$ 60$ over one year, but not in the arm eligible for $\$ 30$. Indeed, interventions with relatively small (conditional) financial payments do not appear to result in measurable reductions in HIV or STI prevalence. Kohler and Thornton (2012) assess an experiment in Malawi that offered a single cash reward of up to $\$ 10$ after one year to individuals who remained HIV negative. The intervention had no measurable effect on HIV status. Finally, Duflo, Dupas and Kremer (2015) find no impact (even in the longer run) on STI from an education subsidy program in Kenya, which subsidized the cost of education for upper primary school students by providing free school uniforms. The education subsidy combined with HIV prevention education focusing on abstinence until marriage, however, resulted in a significant reduction in STI compared to the control group.

Our findings have implications for both policy and research. In both biomedical and behavioral prevention programs, low demand and high costs are often highlighted as constraining factors for successful scale up. Our evidence suggests that lotteries may be an effective method to enhance the demand for safer sexual behavior. Such a lottery program could presumably also be employed to increase the demand for effective biomedical HIV prevention initiatives and could possibly be implemented at lower costs than traditional CCT programs as only winners need to be paid. Moreover, we show that a large share of the individuals that changed their behavior in response to the lottery intervention had a relatively high ex ante risk of getting infected by HIV. Targeting those 
with the highest risk of becoming infected is a key objective in many HIV promotion programs. Such targeting is also important in many other types of prevention programs. Thus, our findings open up new avenues for future research.

The remainder of the paper is organized as follows. Details on the study setting, the research design, and the intervention are presented in section 2. Section 3 reports the main results. Section 4 discusses the implications of the findings as well as study limitations. Section 5 concludes. Additional results are reported in appendix.

\section{Experimental design and data}

\section{A. Study setting}

Lesotho is a small lower-middle-income country with an estimated population of 2.1 million. Poverty is widespread with 43 percent of the population (in 2003) living on less than $\$ 1.25$ a day (World Bank, 2014). Lesotho has one of the highest HIV adult prevalence rate in the world (23.3 percent), with the HIV prevalence rate peaking at 40.5 percent among individuals aged 30-34 (Ministry of Health and Social Welfare and ICF Macro, 2010). Largely due to the HIV/AIDS epidemic, life expectancy at birth is low even by African standards (48 years).

\section{B. Conceptual framework}

We designed a financial incentive program - a lottery - with relatively low expected payments but with high prizes conditional on negative STI test results. As sexually transmitted infections can be viewed as markers for risky sexual behaviors (Crosby et al, 2003; Fishbein and Pequegnat, 2000), our intervention aimed at modifying the trade-off between the benefit and costs of unprotected sex. If individuals' decisions on sexual behavior ignore the health externality of risky sexual behavior, such a transfer program can be justified by the negative externalities generated by a higher number of HIV positive individuals within a society. That is, the (expected) transfer can be viewed as a Pigouvian subsidy aimed at correcting the externality.

The use of lotteries and thus an uncertain return contingent on behavioral change makes lottery incentives different from traditional conditional cash transfer (CCT) programs. Specifically, the expected utility of a lottery with cash prizes depends on individuals' attitude towards monetary risk. If individuals exhibiting risk-loving preferences in monetary gambles are also more risk-loving in other domains, including sexual behavior, lottery incentives may be particularly effective in targeting individuals with the highest risk of HIV/AIDS.

\section{Trial design}

The study was a two-year parallel group randomized trial. It had three separate arms - a control arm with an allocation ratio of 40 percent and two intervention arms - lowvalue lottery and high-value lottery - with an allocation ratio of 30 percent each. In the 
low-value lottery arm individuals were eligible to win lottery prizes worth 500 maloti or approximately $\$ 50$ every four months conditional on being tested negative for two curable STIs (syphilis and trichonomiasis). In the high-value lottery arm individuals were eligible to win lottery prizes of twice that amount (1,000 maloti or approximately $\$ 100)$, again conditionally of testing negative for syphilis and trichonomiasis.

In expected terms, and conditional on being STI negative, the lottery paid $\$ 3.3$ every four months in the low-value lottery group, $\$ 6.6$ in the high-value lottery group and $\$ 4.9$ in the pooled intervention group. In the pooled intervention group, and over the two-year trial period, participants were thus eligible to receive approximately $\$ 30$ in expectation. Both lottery prizes represent a meaningful proportion of household income, especially for women. The study population were primarily shepherds and self-employed workers and data from the Lesotho Labor Force Survey (2008) show that average monthly earning in the informal sector was 235 maloti (approximately \$23.5) for men and 135 maloti (approximately $\$ 13.5$ ) for women.

The choice to condition the incentives on two curable STIs, rather than directly on HIV status, was made for both ethical and epidemiological reasons. First, conditioning incentives on curable STIs allowed individuals testing positive at one round to be eligible to win in the following rounds, if the individual got treatment and remained STI negative. Second, conditioning incentives on curable STIs allowed inclusion of HIV positive individuals in the trial. Risk reduction among HIV positive individuals may have higher impact on HIV transmission in the community than that of HIV negative individuals.

The choice to condition the incentives on syphilis and trichonomiasis status was based on the relatively high prevalence of these two STIs in Lesotho and the fact that they are curable and rapid test technologies are available, practical and affordable. Both STIs have high co-infection rates with HIV (Johnson and Lewis, 2008; Kalichman, Pellowski and Turner, 2011).

Similarly to other conditional cash transfer programs aimed at incentivizing safer sexual behavior, the intervention was designed to offer frequent rewards at short intervals (every 4 months). The short time interval between lottery rounds was intended to bring the benefits of safe sex closer to the present, which may be important if many individuals have high discount rates or a limited horizon (Oster, 2012).

\section{Participants}

Inclusion criteria consisted of males and females, aged 18-32 years, residing in 29 rural and peri-urban villages across 5 districts in Lesotho. Both HIV-positive and HIVnegative individuals were eligible to enroll. Participation was voluntary but a variety of channels were used to maximize the interest in the project and boost participation. ${ }^{4}$

\footnotetext{
${ }^{4}$ Although recruitment was based on voluntary enrollment the sample looks similar to the general population of Lesotho. HIV prevalence rates by age group according to the Lesotho DHS 2009 were: age 15-19: 4.1 percent for women (2.9 percent for men); age 20-24: 24.1 percent for women (5.9 percent for men); age 25-29: 35.4 percent for women (18.4 percent for men). HIV prevalence at baseline in our sample was: age 18-19: 5.3 percent for women (1.1 percent for men); age 20-24: 17.6 percent for women (4.2 percent for men); age 25-29: 29.1 percent for women (15.2 percent for men).
} 
First, a community liaison officer from the implementing NGO visited each participating community before the beginning of the project to garner support from village leaders. Second, media channels were utilized in the form of radio announcements through the most popular local radio stations. Third, posters advertising the project were put up in visible places to inform the communities about the project. Approximately 40 percent of the age-eligible population in the targeted villages volunteered to participate in the study, by coming to the mobile study clinics set up for one week in the centre of the villages. In total, the various procedures yielded a baseline study sample of 3,427 individuals, of which 3,029 (88.4 percent) - the core sample - completed baseline interview and tests for syphilis, trichonomiasis, and HIV.

\section{E. Implementation}

Randomization took place at study mobile clinics in each village separately after baseline interview and STI/HIV testing, with participants selecting one of 10 colored marbles from an opaque bag containing 4 marbles assigning to the control arm, 3 marbles assigning to the low lottery prize arm and 3 marbles assigning to the high lottery prize arm. This highly transparent procedure was considered necessary for acceptability of randomization in the study population.

After completion of the questionnaire and STIs tests, lottery tickets were distributed to all the individuals who tested negative for both syphilis and trichonomiasis in the treatment arms. Individuals in the intervention arms testing positive for any of the two STIs did not receive a lottery ticket. They could, however, continue as study participants and thus become eligible in subsequent rounds. Individuals in the control arm were not eligible for lottery tickets, but all other study procedures were identical between the control and intervention arms. Anyone testing positive for a STI (regardless of arm) was offered counseling and free STI treatment and individuals tested positive for HIV were referred to public health clinics offering AIDS treatment for appropriate follow-up. Individual pre-test and post-test counseling following Lesotho national guidelines was provided to study enrollees at each testing interval.

Lottery draws, organized every four months in each village, were conducted approximately one week after the first interview. Four lottery winners (one male and one female per lottery arm) per village were drawn. ${ }^{5}$

Over two years, we conducted 7 rounds of data collection. At baseline each individual received an ID card with a unique identification number that was used to identify the respondents in subsequent rounds. Each participant received a small in-kind incentive (candles, matches, and washing powder), worth approximately $\$ 3$, as a reward for their participation.

\footnotetext{
${ }^{5}$ Except for the four winners, the identity of individuals included in the lottery draws was not revealed and could not be inferred. The lottery ticket were stored in a closed wooden urn and destroyed after each lottery draw. It was up to the respondents to disclose their status to their partners or peers/neighbors, if they wanted.
} 


\section{F. Measurement}

All participants were tested for HIV, following the Lesotho national testing guidelines, at baseline and months 16, 20 and 24. Participants were tested for syphilis and trichonomiasis at baseline and before each lottery draw.

A baseline and a follow up survey were administered to all participants. The surveys were conducted in private with an enumerator of the same gender as the respondent to mitigate potential reporting biases. The surveys included modules on socioeconomic characteristics, sexual attitudes and behaviors, and knowledge about HIV/AIDS and other sexually transmitted infections. Participants' preferences for risk were measured using a simple multiple price list (MPL) design (Andersen et al. 2008). ${ }^{6}$ In the baseline survey, individuals were presented with a question with 15 decision rows. For each decision row, respondents were asked to choose between a fixed amount, with payments starting at 0 and increasing by 25 maloti (\$2.5) up to 350 maloti (\$35), or a risky lottery with a 50 percent chance to win 500 maloti $(\$ 50)$ or 50 percent chance to receive nothing. We made clear that we were putting them in a hypothetical situation, but that they should try to provide an answer that was as realistic as possible.

We create two measures of risk preferences at baseline. First, we construct an indicator variable ("Risk lover") which takes the value 0 for respondents who preferred a fixed amount of money below the expected value (of 250 maloti) instead of a lottery with 50 percent chance of winning 500 maloti, and 1 otherwise. Second, we follow Andersen et al. (2008) and assume a constant relative risk aversion (CRRA) utility function, $u(y)=$ $\frac{y^{1-r}}{1-r}$, where $y$ is the lottery prize and $r$ is the latent risk coefficient. The point at which respondents switch from the risky to the safe option are then used to deduce bounds on the subject's risk preference $(r){ }^{7}$ We use the midpoints on these bounds as a measure of the participants' preferences for risk.

\section{G. Timing}

Recruitment and baseline data collection started in February, 2010. The lottery trial was stopped after two years, following the protocol. A follow-up study was implemented one year after the intervention ended (February-May, 2013). The time line of the project is described in Figure 1.

Insert Figure 1 here

\section{Results}

\section{Baseline characteristics}

\footnotetext{
${ }^{6}$ The MPL design has been used to elicit risk attitudes by, among others, Holt and Laury (2002) and Tanaka, Camerer and Nguyen (2010).

${ }^{7}$ That is, if the respondent chose the risky option when the safe option paid $s$, but the safe option when safe option paid $s+25$, the bounds are $1-\frac{\ln (2)}{\ln (500)-\ln (s+25)}<r<1-\frac{\ln (2)}{\ln (500)-\ln (s)}$.
} 
Baseline HIV and STI prevalence rates are reported in Table 1 for the core sample (Panel A, column 2). At baseline, 16.7 percent of the study participants tested positive for HIV and 13.5 percent tested positive for any of the two STIs (syphilis and trichonomiasis). ${ }^{8}$ Consistent with data from the 2009 DHS survey in Lesotho, the HIV prevalence rate was significantly higher for females (20.4 percent) than males ( 8.7 percent) and significantly higher for the older compared to the younger age cohorts. Similar differences were observed for STI prevalence.

\section{Insert Table 1 here}

Baseline characteristics by assignment group are further displayed in columns 3-5. ${ }^{9}$ Prevalence rates for HIV, and the two curable STIs, were similar across groups (panel A). The three assignment groups also had similar demographic and socioeconomic characteristics (panel B). Self-reported sexual behavior outcomes (panel C) were also similar on all but two outcomes - self-reported use of a condom during last intercourse and self-reported likelihood that the last partner was HIV infected.

In the last rows of panels $\mathrm{C}$ and $\mathrm{D}$ we report average standardized pre-treatment effects of the four sexual behavior outcomes; i.e., we estimate a seemingly unrelated regression system,

$$
Y=\left[I_{K} \otimes T\right] \beta+v,
$$

where $Y$ is a vector of $K$ related sexual behavior outcomes, $I_{K}$ is a $K$ by $K$ identity matrix, $T$ is a vector of assignment to intervention group(s) indicators and derive an average standardized pre-treatment effect, $\tilde{\beta}=\frac{1}{K} \sum_{k=1}^{K} \frac{\hat{\beta}_{k}}{\hat{\sigma}_{k}}$, where $\hat{\beta}_{k}$ is the point estimate on the treatment indicator in the $k^{\text {th }}$ outcome regression and $\hat{\sigma}_{k}$ is the standard deviation of the control group for outcome $k$ (see Kling et al., 2004; Duflo et al., 2008). We find no significant difference between the treatment groups and the control group using this aggregate measure of self-reported sexual behavior (denoted "Practice safe sex"). Furthermore, as shown in panel D, among HIV negative individuals at baseline - the main sample for the HIV incidence analysis - all four self-reported sexual behavior outcomes - were also similar across assignment groups.

\section{Insert Table 2 here}

The attrition rate was low (Table 2), with 95.4 percent (2888 out if 3029) of the participants surveyed and tested in the last round and 94.6 percent $(2,865$ out of 3,029$)$ of the participants surveyed and tested in the one-year follow-up round. The attrition rate (5-6 percent) was similar across the three assignment groups and not predicted by any of

\footnotetext{
83.9 percent of the respondents were tested positive for syphilis while the prevalence of Trichomoniasis was 10.5 percent. To increase precision, we consider the joint measure of syphilis and trichomoniasis prevalence as the main STI outcome.

${ }^{9}$ In expectations, 30 percent of the sample should have been assigned to the high and low lottery arm, respectively. While the share assigned to the high [low] group is higher [lower] than 0.3 , we cannot reject the null hypotheses that the sample comes from a distribution with means 0.3 for each group (results available upon request).
} 
the baseline socio-demographic characteristics listed in Table 1, panel B, or STI status, except that HIV positive individuals at baseline were more likely to be lost at follow-up (41 of 507 or 8 percent of HIV positive individuals at baseline were lost at follow-up).

\section{STIs as markers for risky sexual behavior}

The main objective of the intervention was to incentivize safer sexual behavior as a route to reducing the spread of HIV. The lottery incentives, however, were tied to STI status. Table A1 in appendix reports correlations between STI status and HIV status and self-reported sexual behavior using baseline data. STI-positive individuals were approximately 2.5 times more likely than STI-negative individuals to be HIV positive at baseline (column 1); i.e., there is a strong positive correlation between STI and HIV status. Columns (2)-(6) show that STI-positive individuals were also more likely to be involved in (self-reported) risky sexual behavior. The average standardized effect ("Practice safe sex") is significantly negative and precisely estimated, providing evidence in favor of the assumption that prevalence of the two STIs can be viewed as a marker for risky sexual behavior.

\section{Average treatment effects: HIV and STI}

To assess the impact of the lottery intervention, we compare mean outcomes after accounting for stratification. That is, we estimate

$$
y_{i j}=\alpha+\beta^{L} T_{i j}^{L}+\beta^{H} T_{i j}^{H}+\eta_{j}+\varepsilon_{i j},
$$

where $y_{i j}$ is a binary variable that for HIV incidence takes value 1 if individual $i$ living in village $j$ became HIV infected over the trial period and 0 otherwise. For HIV [STI] prevalence, $y_{i j}$ takes value 1 if individual $i$ living in village $j$ was HIV [STI] positive at the end of the trial, and 0 otherwise. $T^{L}$ and $T^{H}$ are indicator variables for assignment to the two intervention groups (low and high lottery group, respectively), $\eta_{j}$ are village fixed effects, and $\varepsilon_{i j}$ is an error term. For the main outcome variables, we report both robust standard errors as well as the p-values of tests of the null that treatment has no effect computed using randomization inference. We constructed these p-values using 1000 randomly selected permutations of the randomization allocation. The p-value is then constructed based on the proportion of test statistic values (squared of the estimated coefficients) that are greater than the actual test statistic value. We estimate the effects for the high- and low-value lottery arm and the pooled treatment effect using an OLS model.

\section{Insert Table 3 here}

Table 3 describes the impact of the lottery program on HIV incidence - the primary study outcome. Over the two-year trial period, the HIV incidence rate was reduced by 2.5 percentage points, or 21.4 percent (column 1), leading to a 3.4 percentage points lower HIV prevalence rate at the end of the trial (column 3), in the pooled intervention compared to the control group. Columns (2) and (4) report the effects by intervention group. Relative to the control arm, HIV incidence fell by 3.3 percentage points, or 28 
percent, in the high prize lottery arm, and by half that size, 1.6 percentage points or 14 percent, in the low prize lottery arm. The HIV incidence rates did not differ significantly between the two intervention arms. However, the difference in HIV incidence between the high price lottery arm and the control group was statistically significant while the difference in HIV incidence between the low price lottery arm and the control group was not. ${ }^{10}$

\section{Insert Table 4 here}

In Table 4, we report treatment effects by gender. For HIV incidence, the point estimates for the pooled intervention group and the high value lottery arm were statistically significant in the sample of female participants but not significant in the sample of male participants. However, we cannot reject the equality of treatment effects for female and male participants for the pooled intervention group $(\mathrm{F}$-stat $=2.09$, $\mathrm{p}$-value $=0.15$ for HIV incidence; F-stat $=0.22$, p-value $=0.64$ for HIV prevalence). Overall the differences between male and female participants should be interpreted with caution because the study was not designed to pick up gender specific treatment effects and ex post power calculations show that the study was not powered to detect effects on HIV incidence for men only. ${ }^{11}$

\section{Insert Table 5 here}

Table 5 reports the estimated treatment effects on syphilis and trichonomiasis. Two findings stand out. First, STI prevalence rates fell in all three assignment groups. At baseline, 13.5 percent of the participants were infected by at least one of the two STIs (see Table 1). After two years, STI prevalence in the control group is 3.8 percent. This large reduction in STI prevalence in the control group should be viewed through the lens of the trial protocol. Regular screening and free treatment of the two STIs were provided across all three study groups throughout the trial to participants who were tested positive for STIs. Second, in the intervention groups STI prevalence was essentially zero in both lottery arms $(0.2$ percent in the high lottery arm and 0.5 percent the low lottery arm), implying effect sizes of 89 percent and 82 percent, respectively. Our core sample consists of HIV negative individuals at baseline. However, participation in the project was not conditional on HIV status. Columns (5)-(6) of Table 5 show that the lottery program also affected STI outcomes for the subsample of HIV-positive participants (as measured at baseline). STI prevalence among HIV-positive participants in the control group was 11.2 percent (22/196), while no HIV-positive participants in the intervention arms were tested positive for the two STIs at the end of the trial. Table A4 in the appendix breaks down the STI results by gender. We document large effects for both the group of

\footnotetext{
${ }^{10}$ Adjusted odds-ratios and relative risk ratios are reported in table A2 in appendix. To examine potential bias due to non-random attrition, table A3 in appendix reports Lee bounds estimates. The significant effects for the pooled lottery and the high lottery arms remain intact.

${ }^{11}$ Female and male participants differed in other observables, for example in earnings. The average income earned in the last month (constructed as the sum of household income, wage and non-wage activities) for men is 200 Maloti (about $20 \$$ ) and for women is about 60 Maloti (about $6 \$$ ). The difference in earnings by gender might explain the differential impact on HIV incidence given that lottery prizes are a higher fraction of earnings for women in the sample.
} 
male (significant at the 10 percent-level) and female participants (significant at the 1 percent-level).

\section{Sexual behavior and reproductive health}

Sexual transmission is responsible for the vast majority of new HIV infections, particularly in Sub-Saharan Africa. The risk of getting HIV infected, in turn, can be reduced in several ways, including reducing the number of partners/sexual acts, changing the type of partner, and increasing the use of condoms. In this section we start by studying more closely these behavioral changes.

We focus on four self-reported outcomes: the number of partners in the last four months, whether a condom was used in the last intercourse, if the respondent think his/her last sexual partner was infected by HIV, and whether the last intercourse was extramarital sex. The results are reported in panel A, Table 6.

\section{Insert Table 6 here}

There is evidence of a reduction in risky sexual behavior across all four outcome measures and the average standardized treatment effect on safer sexual behavior, reported in column 5, is statistically significant. ${ }^{12}$ An omnibus randomization test of overall significance (Young, 2016) confirms the result. The estimated treatment effects are small for the number of partner, the use of a condom during last intercourse, and the likelihood, as perceived by the respondent, that the last partner was HIV positive but relatively large and statistically significant for self-reported extramarital sex (which dropped by 23 percent in the treatment relative the control group). Thus, with the caveat that sexual behavior is difficult to measure using traditional survey techniques, the evidence in panel A suggests that the intervention primarily resulted in changes in the type of sexual partner. ${ }^{13}$

The primary objective of the intervention was to reduce HIV incidence. However, a shift towards less risky sexual behavior may also have additional health and socioeconomic benefits. The reduction in the prevalence of syphilis and trichonomiasis, as reported above, is one example. In panel B, Table 6, we assess the effects of two additional reproductive health outcomes: pregnancy and births. As reported in column (6), women in the pooled intervention group were 22.3 percent less likely to have given birth in the last four months or be currently pregnant. Columns (7)-(8) split the sample on whether or not the woman live in a long-term relationship (here labeled as married versus unmarried). Pregnancies among single women are more likely to be unwanted and due to unprotected sex with a non-regular partner. The results on the split sample suggest that the effect for single women (a reduction by 31 percent) is almost twice as large and more precisely estimated compared to the effect for married women. ${ }^{14}$

\footnotetext{
12 "Practice safe sex" is the average standardized effect of the four individual indicators in panel A, reversing the sign of "Number of partners last 4 months", "High likelihood last partner HIV+", and "Extramarital sex". Here we are considering individuals who had sex in the last 4 months, but results are very similar when also including respondents who did not have sexual intercourse in the last 4 months.

${ }^{13}$ Recall and social desirability biases in self-reported data on sexual behavior are a general concern, and especially so when the data are collected within the context of a project involving counseling and testing, as is the case here (Strauss and Thomas, 1998; Powers et al., 2008; Boily et al., 2009; Corno and De Paula, 2014).

${ }^{14}$ Equality of treatment effects for unmarried and married women can not be rejected ( $p$-value $=0.61$ ).
} 
Taken together, the findings on self-reported sexual behavior, as well as other reproductive health outcomes, show that behavior changed in response to the intervention and that these sexual behavioral changes also resulted in benefits apart from those we primarily targeted.

\section{Heterogeneous effects: Preferences for risk}

A better understanding of how to strategically target social behavioral programs to groups at higher risk of infection is often raised as a priority by policy makers. Apart from specific groups (commercial sex workers for example), however, little is known about how to identify and target those at high risk, in particularly in a population at large (UNAIDS, 2013). If more risk-loving individuals are also more risk-prone in other domains, including sexual behavior, the introduction of lotteries into an otherwise standard financial incentive program may be one way to better target those at higher risk of getting infected by HIV. In this section we investigate whether there is evidence in favor of such a mechanism.

Participants' preferences for risk at baseline were measured using a simple multiple price list (MPL) design as described in section 2.6. Table A5 in appendix replicates the 15 tasks presented to participants at baseline and the implied bounds on the latent risk coefficient $r$. The midpoints on these bounds are used to measure the respondents' preferences $(r)$ for risk. In total, 60 percent of the respondents switched from choosing the risky lottery to the safe option; i.e. for these respondents we can deduce bounds on $r$. The remaining 40 percent of the respondents either choose the safe lottery for all 15 options being presented, including the first option where the safe option paid 0 (the expected value in the risky lottery was always 250 maloti), or choose the risky lottery for all 15 options presented, which implies a risk coefficient $r<-0.94$. While we cannot rule out that some individuals place a non-pecuniary cost of participating in a lottery, and thus prefer nothing as compared to a chance of winning, or that some individuals are highly risk loving, these choices are more likely to be a product of lack of comprehension of the MPL design. Thus we restrict the sample to the sub-sample of switchers (for which we can deduce $r$ ). As a robustness test, however, we reestimate all core specifications with respondents always choosing the safe option coded as risk-averse (0) and respondents always choosing the risky option coded as risk-lovers (1).

Summary statistics for our two measures of risk preferences at baseline are provided in Table 1, Panel B. 35 percent of the sample are "risk lover", that is they prefer a lottery with 50 percent chance of winning 500 maloti compared to a fixed amount of money greater or equal to its expected value (of 250 maloti). The risk coefficient $(r)$ is 0.245 on average, which is lower than that found in Holt and Laury (2002) for university students in the US ( $r=0.27$ on average) and Harrison et al. (2010) for poor subjects in Ethiopia, India and Uganda ( $r=0.54$ on average), but higher than those reported in Lammers and van Wijnbergen (2007) for students in South Africa ( $r=0.16$ on average) and in Tanner et al. (2005) for Nigerian subjects ( $r=0.15$ on average). Both measures are well balanced across treatment arms at baseline.

As individuals that exhibit risk-loving preferences in a monetary gamble may not be risk-loving in other domains, especially when it comes to sexual behavior, we start by 
investigating whether our measure of risk ("Risk lover") has bearing on risky sexual behavior. Table A6 in the appendix compares risk-loving and risk-averse individuals on observable characteristics at baseline. Risk-averse and risk-loving individuals had similar demographic and socioeconomic characteristics (panel B), but risk-loving participants were more likely to be HIV positive. Risk-loving participants were also less likely to report that they practice safe sex (panel C) and have on average a higher STI prevalence rate (statistically significant at the 10 percent-level) at baseline compared to risk-averse participants.

Do risk-loving individuals respond differently than risk-averse individuals to the lottery program? In Table A7, we start by regressing HIV incidence on risk attitudes separately for each assignment group. Risk-lovers were more than twice as likely to become infected with HIV over the trial period in the control group (column 1). That is, although risk-lovers account for about one-third of the sample, they still account for more than half of the new HIV infections in the control group. In the pooled intervention group, on the other hand, the share of individuals infected by HIV during the trial period is approximately the same in the group of risk-loving and risk-averse individuals (column 2). Similar results are obtained when using the continuous risk coefficient $r$ (columns 3-4).

\section{Insert Table 7 here}

Table 7 uses the core sample and an interaction term between the pooled intervention group and the risk-lover indicator. HIV incidence was 11 percentage points lower for risk-loving individuals in the intervention relative the control group (column 1). In both the intervention and control group, HIV incidence among risk-averse is approximately the same. Column (2) shows that the relationship between risk aversion and risky sexual behavior remain intact when we replace the indicator variable of risk attitudes with the continuous risk coefficient $r$.

\section{Insert Figure 2 here}

The relationship between risk aversion and HIV incidence in the intervention and the control group is graphically illustrated in Figure 2, using a nonparametric Fan local regression method. Consistent with the findings reported in columns (1)-(2), the data suggest that the lottery program lowered HIV incidence in the intervention group by making risk-loving individuals behave similarly to risk-averse individuals in terms of their sexual behavior; i.e., the effect we observe appears to be driven primarily by the risk-loving participants. The treatment effect for this sub-group is quantitatively large: individuals with a deduced risk aversion coefficient one-standard deviation below the mean were 53 percent less likely to have been infected by HIV during the trial period in the intervention relative to the control group.

Participants' preferences for risk are not randomly assigned. Thus a concern with the results reported in columns (1)-(2) is that the treatment effects for risk-loving individuals reflect differences in demographic and socioeconomic characteristics across participants, rather than truly stronger response to the lottery incentives for this sub-group. To partly address this concern we add the full set of baseline demographic and socioeconomic 
characteristics (listed in Table 1, panel B) and STI status at baseline as controls as well as interactions between assignment to intervention and all additional covariates in columns (3)-(4). The results remain unchanged, suggesting that differences in demographic and socioeconomic characteristics and STI status at baseline do not drive the results reported in columns (1)-(2).

In Table A8 in appendix, we expand the sample by also including individuals that choose either the safe or risky options for all values of the safe payment in the MPL question. The main results remain intact, albeit less precisely estimated. If the noswitching behavior is driven by a lack of comprehension of the MPL design, the inclusion of the non-switchers will likely attenuate the true effect. The results in Table A8 are consistent with this interpretation.

Columns (5)-(8) in Tables 7 and A7 show that the results remain broadly intact when using STI prevalence rather than HIV incidence as dependent variable (although the result is only significant for the continuous risk coefficient $r$ ). In both the group of risk-averse and the group of risk-loving individuals we observe large reductions in the prevalence of any of the two STIs in the intervention as compared to the control group. All 645 risk-averse individuals and 334 out of 337 risk-lovers (99.1 percent) in the pooled intervention group were tested STI negative at the end of the project period. In the control group 5.2 percent (12 out of 232) of the risk-loving individuals and 2.2 percent (9 out of 406) of the risk-averse individuals were tested STI positive at the end of the trial (see table A7). For the group of risk-loving individuals this corresponds to a reduction in STI prevalence of 83 percent.

\section{Longer run effects}

The results reported so far were limited to a 24 months program with recurrent village level lotteries every fourth months, and cannot address the sustainability of the decline of HIV incidence over a longer period, particularly after the lottery program has been discontinued. Nor can the results address the possibility of adverse consequences to the extent that extrinsic incentives may reduce long-term intrinsic motivation to engage in safe sexual behaviors after incentives have been withdrawn. On the other hand, work on sexual behavior in Sub-Saharan Africa have highlighted various cultural barriers to changing behavior (e.g., fatalism, low levels of female bargaining power), and it is possible that the financial scheme considered here helps overcome these adverse cultural constraints.

\section{Insert Table 8 here}

To assess the longer run effects, a follow-up study was implemented one year after the intervention ended. The survey was not announced in advance. In the follow-up study we re-interviewed and re-tested the participants that were screened at the end of the intervention. Table 8 summarizes the main findings in the first year following the intervention. As evident, HIV incidence rates were similar across assignment groups in the year following the trial (column 1). That is, there is no evidence of adverse reactions or consequences in the intervention relative to the control group, at least based on data one year after the intervention ended. As a result, HIV prevalence remains significantly 
different between the pooled intervention and control group (column 2) one year after the intervention ended. We also find lower STI prevalence in the treatment compared to the control group and the effect is statistically significant. Self-reported practice of safe sexual behavior one year later has decreased slightly compared to endline ( $0.027 \mathrm{vs}$ 0.060 ) and is no longer significant. Similarly, the effect on pregnancy is not statistically significant. ${ }^{15}$

\section{Discussion}

\section{A. Cost-effectiveness}

The lottery program reduced risky sexual behavior at relatively low costs. ${ }^{16}$ We calculate that the cost of averting a primary HIV infection in the Lesotho trial was $\$ 882$ based on cash payments only (cash rewards of $\$ 30$ per year or \$59.4 in PPP in the pooled intervention group). If we add costs related to STI testing and program administration, the cost per infection averted is estimated at $\$ 3,324$. As a reference, Kohler and Thornton (2012) assessed an experiment in Malawi that offered a single cash reward of up to $\$ 10$ (\$26.5 in PPP terms) after one year to individuals who remained HIV negative. The intervention had no measurable effect on HIV status; i.e., infinite cost per HIV infection averted. Baird et al. (2012) evaluated an intervention, also in Malawi, targeting human capital formation as an alternative HIV prevention strategy. They found that 15 monthly cash transfers of $\$ 10$ ( $\$ 24.5$ in PPP) per household and conditional on school attendance for adolescent girls led to a significant reduction in HIV prevalence after 18 months. The cost per HIV infection averted was $\$ 8,333$ based on cash payments only, and $\$ 12,500$ adding the administrative costs. Another conditional cash transfer trial, in Tanzania, that shares a number of similarities to the Lesotho trial (financial incentives tied to periodic screening of STIs, free STIs treatment of all trial participants, similar inclusion criteria, and almost identical prevalence rates for any of syphilis and trichomonas at baseline) but where the cash rewards were higher and paid with certainty, report STIs prevalence as the key outcome variable (de Walque et al., 2012). They document a reduction in STIs prevalence in the treatment arm where participants were eligible for $\$ 60$ ( $\$ 178.9$ in PPP) but find no measurable effect on STIs prevalence in the pooled intervention group where participants were eligible for $\$ 40$ ( $\$ 119.20$ in PPP) on average. The study was not powered to directly examine HIV conversion, thus implications for HIV prevention remain speculative. The cost of averting a STIs infection at the end of the study period was $\$ 2500$ based on cash payments only and $\$ 8,164$ including the STI testing and administrative costs. The estimated cost of averting an STI infection in the Lesotho trial was significantly lower: \$645 based on cash payments, \$2,943 including STI testing and administration.

\footnotetext{
${ }^{15}$ Results available upon request.

${ }^{16}$ A comparison of cost-effectiveness across studies should be considered as one input among others in assessing policy, especially because some interventions may create benefits beyond reducing the risk of HIV infections and because some interventions may be more context-dependent and sensitive to small differences in program design.
} 


\section{B. Structured speculation}

We show that a financial incentive program designed in the form of a lottery with low expected payments but with relatively high payments for lottery winners resulted in significant reduction in HIV incidence. In this section, we discuss how generalizable this finding is, following Banerjee, Chassang and Snowberg (2017). We ask two questions: (1) Would the certainty equivalent of the conditional lottery incentive program cause a similar reduction in HIV incidence? (2) For what types of health behaviors and contexts would we expect the same results to obtain?

(1) Would the certainty equivalent of the conditional lottery incentive program cause a similar reduction in HIV incidence?

There are at least two reasons for why a conditional financial incentive program where participants are rewarded with a chance to win would result in larger reduction in HIV incidence compared to the certainty equivalent of such a lottery program.

First, there is growing evidence from psychology and behavioral economics that people tend to overestimate the probability of rare events, and as a result may prefer a small chance at a large reward to a small reward for sure (Kahneman and Tversky, 1979, Kahneman, 2011, Barberis, 2013). If so the perceived return from participating in a lottery may be higher than the return from an incentive program that pays the expected return with certainty; i.e., conditional payments in the form of a lottery could make the incentive scheme more high-powered compared to the certainty equivalent.

Second, when the intention is to reduce risky behavior, as is the case here, and in a population with heterogeneous preferences for risk, lottery incentives may better target higher-risk individuals; i.e. individuals at higher risk of HIV/AIDS in our setting. A necessary condition for this to be the case is that individuals exhibiting risk-loving preferences in monetary gambles are also more risk-loving in the domain being incentivized (here sexual behavior). If so, more risk-loving individuals may be more likely to change behavior when faced with an incentive program with a chance to win, simply because the utility value of participating in such a lottery is higher than for more risk-averse individuals.

Our experiment was not designed to test these mechanisms, nor to compare lotteries with the certainty equivalent. However, two pieces of evidence suggest that one or both of these mechanisms are indeed at play.

First, other HIV prevention projects based on conditional incentives with certain payments and with roughly the certainty equivalent of the lottery program assessed here have not resulted in significant reductions in the prevalence of sexually transmitted infections in other low-income countries (see Kohler and Thornton, 2012; and de Walque, et al., 2012). That is, and contrary to what we find, low powered incentive schemes in expected utility terms, as is the case here, paid using fixed amounts conditional on testing negative for markers of risky sexual behaviors, have not been shown to be effective. Second, the results reported in Table A7 indicate that, at baseline, risk-loving participants, as defined by their answers to hypothetical monetary gambles, were more likely to be HIV positive and STI positive and less likely to report safer sexual behaviors compared to risk-averse 
participants; i.e., there is a positive correlation between risk-taking in monetary gambles and risky sexual behavior. Moreover, the results shown in Table 7 and Table A8 further suggest that individuals who exhibited risk-loving preferences at baseline were more responsive to the intervention and constitute a large share of the respondents that changed their measured behavior in response to the lottery incentives - a result consistent with the hypothesis that the lottery program targeted those at higher risk of HIV/AIDS.

(2) For what types of health behaviors and contexts would we expect the same results to obtain?

Low demand for effective health prevention methods are observed in many countries. How to effectively encourage preventive health behaviors is however less clear. If individuals systematically overweight low probabilities, or if participation in a lottery yield direct non-pecuniary rewards, for example by offering participants an element of entertainment or fun, lotteries could be used as a complimentary tool to boost uptake. Such a prevention scheme is likely to be particularly promising in programs where preventive health behaviors are relatively easy to measure, such as, for example, programs to encourage uptake of immunization and antenatal care visits. Lottery incentives could also be introduced to increase the demand for other HIV prevention methods, such as HIV screening and male circumcision, where low demand has been identified as a key barrier for successful scale-up. Importantly, a lottery program can presumably also be implemented at lower costs than traditional CCT programs as only winners need to be paid. Permutations of the design, such as only testing winners, could further reduce costs. It could also increase the scalability and applicability of the lottery incentives, to include uptake of various prevention goods, such as bednets.

Our findings suggest that lottery-based incentives may be especially attractive when the aim is to incentivize behavioral changes among risk-loving individuals. Importantly, however, this hypothesis hinges on the assumption that the behaviors causing the health risks, whether it is unsafe sex, smoking, or drug consumption, are particularly common among risk-loving individuals - an assumption that may be difficult to assess ex-ante. Here, however, we do provide suggestive evidence of that being the case. If this holds true in other settings, lottery incentives, properly designed, could, for example, be used to encourage uptake of various promising biomedical prevention interventions, such as pre-exposure prophylaxis to reduce HIV infection risks, which in most settings would require that individuals at high risk can be identified and reached.

\section{Study limitations}

The lottery intervention may have influenced outcomes through other channels than the ones we stress above. For example, a public lottery event every four months could have highlighted the role of prevention in the community. This channel, though, presumably influenced all study participants, independent of assignment arm. Moreover, the experimental evidence on traditional prevention programs (for example risk-reduction counseling, condom promotion, referral and treatment for sexually transmitted infections) have not documented significant effects on HIV incidence. 
A large, albeit uncertain, payment may also be preferable to a lower but certain payment flow if consumers face saving constraints and cannot purchase an indivisible good out of current income. However, this mechanisms cannot account for the heterogeneous results we present, unless less risk-averse individuals also are more likely to face saving constraints.

Finally, our design, where assignment to treatment was randomized within villages, does not allow us to estimate spillover effects. Such spillover effects are likely important and correctly estimating them is crucial to properly assess cost-effectiveness. But it would also require a design that randomize treatment at a more aggregate level. As a reduced transmission risk would affect participants in both the intervention and the control group, the treatment estimates we report in the paper do not capture the indirect spillover effects of a lower transmission risk among (all) participants.

\section{Conclusions}

The extremely high social and economic cost of the HIV/AIDS epidemic motivates the continued search for innovative and cost-effective prevention approaches. In this paper we investigate the effects of introducing a gamble into an otherwise standard financial incentive program. To the best of our knowledge, this is the first large scale randomized trial that assesses the impact of lottery as an HIV prevention tool. We find that the lottery program resulted in a significant reduction in HIV incidence. We further show that a large share of the individuals that changed their measured behavior in response to the intervention exhibited risk-loving preferences at baseline. This finding is consistent with the hypothesis that lotteries are more valued by individuals willing to take risks. Thus, our findings provide suggestive evidence that lotteries can be used to target groups at higher risks of getting HIV infected.

\section{REFERENCES}

Andersen, Steffen, Glenn W. Harrison, Morten I. Lau, and E. Elisabet Rutstrom. 2008. "Eliciting Risk and Time Preferences." Econometrica 76, no. 3: 583-618.

Auvert, Bertran, Dirk Taljaard, Emmanuel Lagarde, Joelle Sobngwi-Tambekou, Rémi Sitta, and Adrian Puren. 2005. "Randomized, Controlled Intervention Trial of Male Circumcision for Reduction of HIV Infection Risk: The ANRS 1265 Trial." PLOS Medicine 2, no. 11: e298.

Bailey, Robert C., Stephen Moses, Corette B. Parker, Kawango Agot, Ian Maclean, John N. Krieger, Carolyn F. M. Williams, Richard T. Campbell, and Jeckoniah O. NdinyaAchola. 2007. "Male Circumcision for HIV Prevention in Young Men in Kisumu, Kenya: A Randomised Controlled Trial." The Lancet 369, no. 9562: 643-656.

Baird, Sarah, Richard Garfein, Craig T. McIntosh, and Berk Özler. 2012. "Effect of a Cash Transfer Programme for Schooling on Prevalence of HIV and Herpes Simplex 
Type 2 in Malawi: A Cluster Randomized Trial." The Lancet 379, no. 9823: 13201329.

Banerjee, Abhijit V., Sylvain Chassang, and Erik Snowberg. 2017. "Decision Theoretic Approaches to Experiment Design." Handbook of Field Experiments 1, edited by Esther Duflo, and Abhijit Banerjee, 141-174. Amsterdam and New York: North Holland.

Barberis, Nicholas C. 2013. "Thirty Years of Prospect Theory in Economics: A Review and Assessment." Journal of Economic Perspectives 27, no. 1: 173-195.

Boily, Marie-Claude, Rebecca F. Baggaley, Lei Wang, Benoit Masse, Richard G. White, Richard J. Hayes, and Michel Alary. 2009. "Heterosexual Risk of HIV-1 Infection per Sexual Act: Systematic Review and Meta-Analysis of Observational Studies." The Lancet Infectious Diseases 9, no. 2: 118-129.

Corno, Lucia, and Áureo De Paula. 2014. "Risky Sexual Behavior: Biological Markers and Self-Reported Data." CEPR Discussion Paper, no. DP10271.

Crosby, Richard A., Ralph J. DiClemente, Gina M. Wingood, Delia Lang, and Kathy F. Harrington. 2003. "Value of Consistent Condom Use: A Study of Sexually Transmitted Disease Prevention among African American Adolescent Females." American Journal of Public Health 93, no. 6: 901-902.

De Walque, Damien, William H. Dow, Rose Nathan, Ramadhani Abdul, Faraji Abilahi, Erick Gong, Zachary Isdahl et al. 2012. "Incentivising Safe Sex: A Randomised Trial of Conditional Cash Transfers for HIV and Sexually Transmitted Infection Prevention in Rural Tanzania." British Medical Journal Open 2, no. 1: e000747. doi:10.1136/bmjopen-2011-000747.

Duflo, Esther, Pascaline Dupas, and Michael Kremer. 2015. "Education, HIV, and Early Fertility: Experimental Evidence from Kenya." American Economic Review 105, no. 9: 2757-2797.

Duflo, Esther, Rachel Glennerster, and Michael Kremer. 2008. "Using Randomization in Development Economics Research: A Toolkit." In Handbook of Development Economics 4, edited by T. Paul Schultz, and John Strauss, 3895-3962. Amsterdam and New York: North Holland.

Fishbein, Martin, and Willo Pequegnat. 2000. "Evaluating AIDS Prevention Interventions using Behavioral and Biological Outcome Measures." Sexually Transmitted Diseases 27, no. 2: 101-110.

Geffen, Nathan. 2011. "What Does Science Show About Incentives for HIV Testing?" Quackdown, December 13, 2011. http://www.quackdown.info/article/whatdoes-science-show-about-incentives-hiv-testing/

Gray, Ronald H., Godfrey Kigozi, David Serwadda, Frederick Makumbi, Stephen Watya, Fred Nalugoda, Noah Kiwanuka et al. 2007. "Male Circumcision for HIV Prevention in Men in Rakai, Uganda: A Randomised Trial." The Lancet 369, no. 9562: 657-666. 
Haisley, Emily, Kevin G. Volpp, Thomas Pellathy, and George Loewenstein. 2012. "The Impact of Alternative Incentive Schemes on Completion of Health Risk Assesments." American Journal of Health Promotion 26, no. 3: 184-188.

Harrison, Glenn W., Steven J. Humphrey, and Arjan Verschoor. 2010. "Choice under Uncertainty: Evidence from Ethiopia, India and Uganda." The Economic Journal 120, no. 543: 80-104.

Holt, Charles A., and Susan Laury. 2002. "Risk Aversion and Incentive Effects." The American Economic Review 92, no. 5: 1644-1655.

Johnson, Leigh F., and David A. Lewis. 2008. "The Effect of Genital Tract Infections on HIV-1 Shedding in the Genital Tract: A Systematic Review and Meta-Analysis." Sexually Transmitted Diseases 35, no. 11: 946-959.

Kahneman, Daniel, and Amos Tversky. 1979. "Prospect Theory: An Analysis of Decision under Risk." Econometrica 47, no. 2: 263-291.

Kahneman, Daniel. 2011. "Thinking, Fast and Slow." New York: Farrar, Straus, and Giroux.

Kalichman, Seth C., Jennifer Pellowski, and Christina Turner. 2011. "Prevalence of Sexually Transmitted Co-Infections in People Living with HIV/AIDS: Systematic Review with Implications for using HIV Treatments for Prevention." Sexually Transmitted Infections 87, no. 3: 183-190. doi:10.1136/sti.2010.047514.

Keating, Joshua E. 2013. "The Nudgy State." Foreign Policy: The Global Magazine of News and Ideas, January 2, 2013. http://foreignpolicy.com/2013/01/02/the-nudgystate/.

Kimmel, Stephen E., Andrea B. Troxel, Benjamin French, George Loewenstein, Jalpa Doshi, Todd E. H. Hecht, Mitchell Laskin, Colleen M. Brensinger, Chris Meussner, and Kevin G. Volpp. 2016. "A Randomized Trial of Lottery-Based Incentives and Reminders to Improve Warfarin Adherence: The Warfarin Incentives (WIN2) Trial." Pharmacoepidemiol Drug Safety 25, no. 11: 1219-1227. doi:10.1002/pds.4094.

Kling, Jeffrey R., Jeffrey B. Liebman, Lawrence F. Katz, and Lisa Sanbonmatsu. 2004. "Moving to Opportunity and Tranquility: Neighborhood Effects on Adult Economic Self-Sufficiency and Health from a Randomized Housing Voucher Experiment." KSG Working Paper, no. RWP04-035.

Kohler, Hans-Peter, and Rebecca L. Thornton. 2012. "Conditional Cash Transfers and HIV/AIDS Prevention: Unconditionally Promising?" World Bank Economic Review 26, no. 2: $165-190$.

Lammers, Judith, and Sweder van Wijnbergen. 2007. "HIV/AIDS, Risk Aversion and Intertemporal Choice." Tinbergen Institute Discussion paper, no. 2007-098/1. 
Lee, David S. 2009. "Training, Wages, and Sample Selection: Estimating Sharp Bounds on Treatment Effects." The Review of Economic Studies 76, no. 3: 1071-1102.

Lesotho Labour Force Survey. 2008. "Lesotho Integrated Labour Force Survey." Ministry of Finance and Development Planning, Bureau of Statistics, Maseru, Lesotho.

McCoy, Sandra I., Rugare A. Kangwende, and Nancy S. Padian. 2010. "Behavior Change Interventions to Prevent HIV Infection among Women Living in Low and Middle Income Countries: A Systematic Review." AIDS and Behavior 14, no. 3: 469-482.

Ministry of Health and Social Welfare and ICF Macro. 2010. "Lesotho Demographic and Health Survey 2009." Maseru, Lesotho.

Oster, Emily. 2012. "HIV and Sexual Behavior Change: Why Not Africa?" Journal of Health Economics 31, no. 1: 35-49.

Padian, Nancy S., Sandra I. McCoy, Jennifer E. Balkus, and Judith N. Wasserheit. 2010. "Weighing the Gold in the Gold Standard: Challenges in HIV Prevention Research." AIDS 24, no. 5: 621-635.

Powers, Kimberly A., Charles Poole, Audrey E. Pettifor, and Myron S. Cohen. 2008. "Rethinking the Heterosexual Infectivity of HIV-1: A Systemic Review and MetaAnalysis." The Lancet Infectious Diseases 8, no. 9: 553-563.

Stitzer, Maxine L., Tiffany Polk, Sarah Bowles, and Thomas Kosten. 2010. "Drug Users' Adherence to a 6-month Vaccination Protocol: Effects of Motivational Incentives." Drug and Alcohol Dependence 107, no. 1: 76-79.

Strauss, John, and Duncan Thomas. 1998. "Health, Nutrition, and Economic Development." Journal of Economic Literature 36, no. 2: 766-817.

Tanaka, Tomomi, Colin F. Camerer, and Quang Nguyen. 2010. "Risk and Time Preferences: Linking Experimental and Household Survey Data from Vietnam." American Economic Review 100, no. 1: 557-571.

Tanner, Mariah., Jayson Lusk, and Wallace Tyner. 2005. "A Multidimensional Investigation of Economic Behavior in Four Countries". Unpublished Manuscript, Purdue University.

UNAIDS. 2013. "AIDS epidemic update: November 2013." UNAIDS, November 2013. Geneva, Switzerland.

UNAIDS. 2016. "Fact Sheet 2016." UNAIDS, 2016. Geneva, Switzerland. www.unaids.org.

Volpp, Kevin G., Leslie K. John, Andrea B. Troxel, Laurie Norton, Jennifer Fassbender, and George Loewenstein. 2008a. "Financial Incentive-Based Approaches for Weight Loss: A Randomized Trial." Journal of the American Medical Association 300, no. 22: 2631-2637. 
Volpp, Kevin G., George Loewenstein, Andrea B. Troxel, Jalpa Doshi, Maureen Price, Mitchell Laskin, and Stephen E. Kimmel. 2008b. "A Test of Financial Incentives to Improve Warfarin Adherence." BMC Health Services Research 8, no. 1: 272.

World Bank. 2014. Data retrieved from World Development Indicators (WDI) Online database.

Young, Alwyn. 2016. "Channelling Fisher: Randomization Tests and the Statistical Insignificance of Seemingly Significant Experimental Results". Unpublished Manuscript, London School of Economics.

Insert Appendix tables A1-A8 here 